\title{
Solid-Phase Microextraction Enables Isolation of BRAF V600E Circulating Tumor DNA from Human Plasma for Detection with a Molecular Beacon Loop-Mediated Isothermal Amplification Assay
}

Marcelino Varona, Derek R. Eitzmann, Darshna Pagariya, Robbyn K. Anand, and Jared L. Anderson*

Department of Chemistry, Iowa State University, Ames, Iowa 50011, United States

\section{Table of Contents}

Table S1

Figure S1

Figure S2

Figure S3

Figure S4

Figure S5

Figure S6

Figure S7

Figure S8 
Table S1. DNA sequences, primers, and probes used in this study.

\begin{tabular}{|c|c|}
\hline 280 bp sequence & $\begin{array}{l}\text { 5'- } \\
\text { GGATGTGTCTGCGGCGTTTTATCATCTTCCTCTTCATCCTGCTGCTATGC } \\
\text { CTCATCTTCTTGTTGGTTCTTCTGGACTATCAAGGTATGTTGCCCGTTTGT } \\
\text { CCTCTAATTCCAGGATCATCAACAACCAGCACCGGACCATGCAAAACCT } \\
\text { GCACAACTCCTGCTCAAGGAACCTCTATGTTTCCCTCATGTTGCTGTACA } \\
\text { AAACCTACGGACGGAAACTGCACCTGTATTCCCATCCCATCATCTTGGG } \\
\text { CTTTCGCAAGTAACCTATGGGAGTGGGCCTC-3' }\end{array}$ \\
\hline qPCR Primers & $\begin{array}{l}\text { Forward-5'- } \\
\text { GGATGTGTCTGCGGCGTTTT-3' Reverse- 5'- } \\
\text { GAGGCCCACTCCCATAGGTT-3 }\end{array}$ \\
\hline Wild type BRAF & $\begin{array}{l}\text { 5'- } \\
\text { TATATTTCTTCATGAAGACCTCACAGTAAAAATAGGTGATTTTGGTCTAGC } \\
\text { TACAGTGAAATCTCGATGGAGTGGGTCCCATCAGTTTGAACAGTTGTCTG } \\
\text { GATCCATTTTGTGGATGGTAAGAATTGAGGCTATTTTTCCACTGATTAAAT } \\
\text { TTTTGGCCCTGAGATGCTGCTGAGTTAC } \\
\text { TAGAAAGTCATTGAAGGTCTCAACTATAGT-3' }\end{array}$ \\
\hline Mutant V600E BRAF & $\begin{array}{l}\text { 5'- } \\
\text { TATATTTCTTCATGAAGACCTCACAGTAAAAATAGGTGATTTTGGTCTAGC } \\
\text { TACAGAGAAATCTCGATGGAGTGGGTCCC } \\
\text { ATCAGTTTGAACAGTTGTCTGGATCCATTT } \\
\text { TGTGGATGGTAAGAATTGAGGCTATTTTTCCACTGATTAAATTTTTGGCC } \\
\text { CTGAGATGCTGCTGAGTTACTAGAAAGTCATTGAAGGTCT } \\
\text { CAACTATAGT-3' }\end{array}$ \\
\hline BRAF_FIP & 5'-TCCAGACAACTGTTCAAACTGATGGTGATTTTGGTCTAG-3' \\
\hline BRAF_BIP & 5'-TTGTGGATGGTAAGAATTGAGGC-TTTCTAGTAACTCAGCAGCA-3' \\
\hline BRAF_F3 & 5'-TCTTCATGAAGACCTCACAG-3' \\
\hline BRAF_B3 & 5'-CTATAGTTGAGACCTTCAATGAC-3' \\
\hline BRAF_LB & 5'-TCCACTGATTAAATTTTTGGCCCTG-3 \\
\hline MB1 & 5'IABkFQ-GGGACCCACTCCATCGAGATTTCAGGGTCCC-FAM-3' \\
\hline MB2 & 5'-FAM-CGCGCCCACTCCATCGAGATTTCACTGGCGCG-IABKFQ-3' \\
\hline MB2-MUT & 5'-HEX-CGCGCCCACTCCATCGAGATTTCTCTGGCGCG-Dabcyl-3' \\
\hline MB2 Complement & 5'-CAGTGAAATCTCGATGGAGTGGG-3' \\
\hline MB2 Mismatch & 5'-CAGAGAAATCTCGATGGAGTGGG-3' \\
\hline $30 \mathrm{nt}$ probe & 5'-FAM-CCGCCCACTCCATCGAGATTTCACTGGCGG-IABKFQ-3' \\
\hline $28 \mathrm{nt}$ probe & 5'-FAM-CGCCCACTCCATCGAGATTTCACTGGCG-IABKFQ-3' \\
\hline $23 \mathrm{nt}$ probe & 5'-FAM-CCCACTCCATCGAGATTTCACTG-IABKFQ-3' \\
\hline
\end{tabular}

Bolded letter denotes the location of the BRAF V600E mutation 

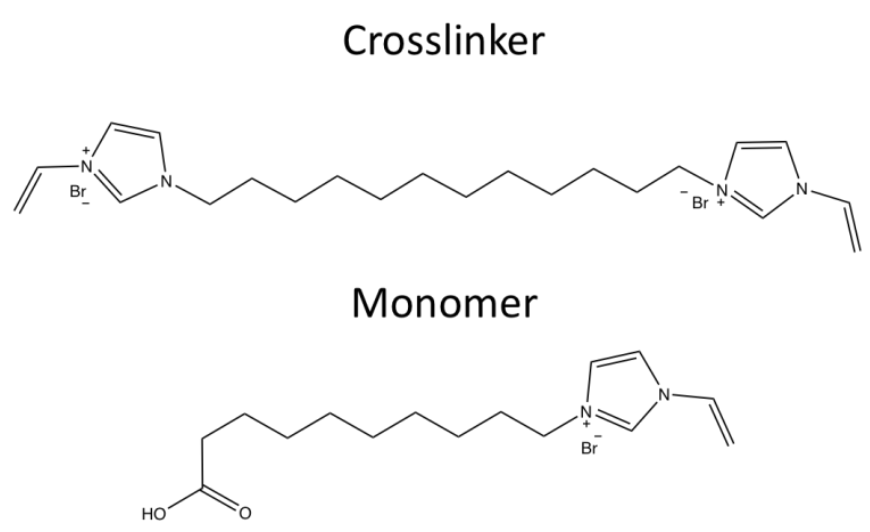

Figure S1. Chemical composition of the PIL-based sorbent coating used in the study. 


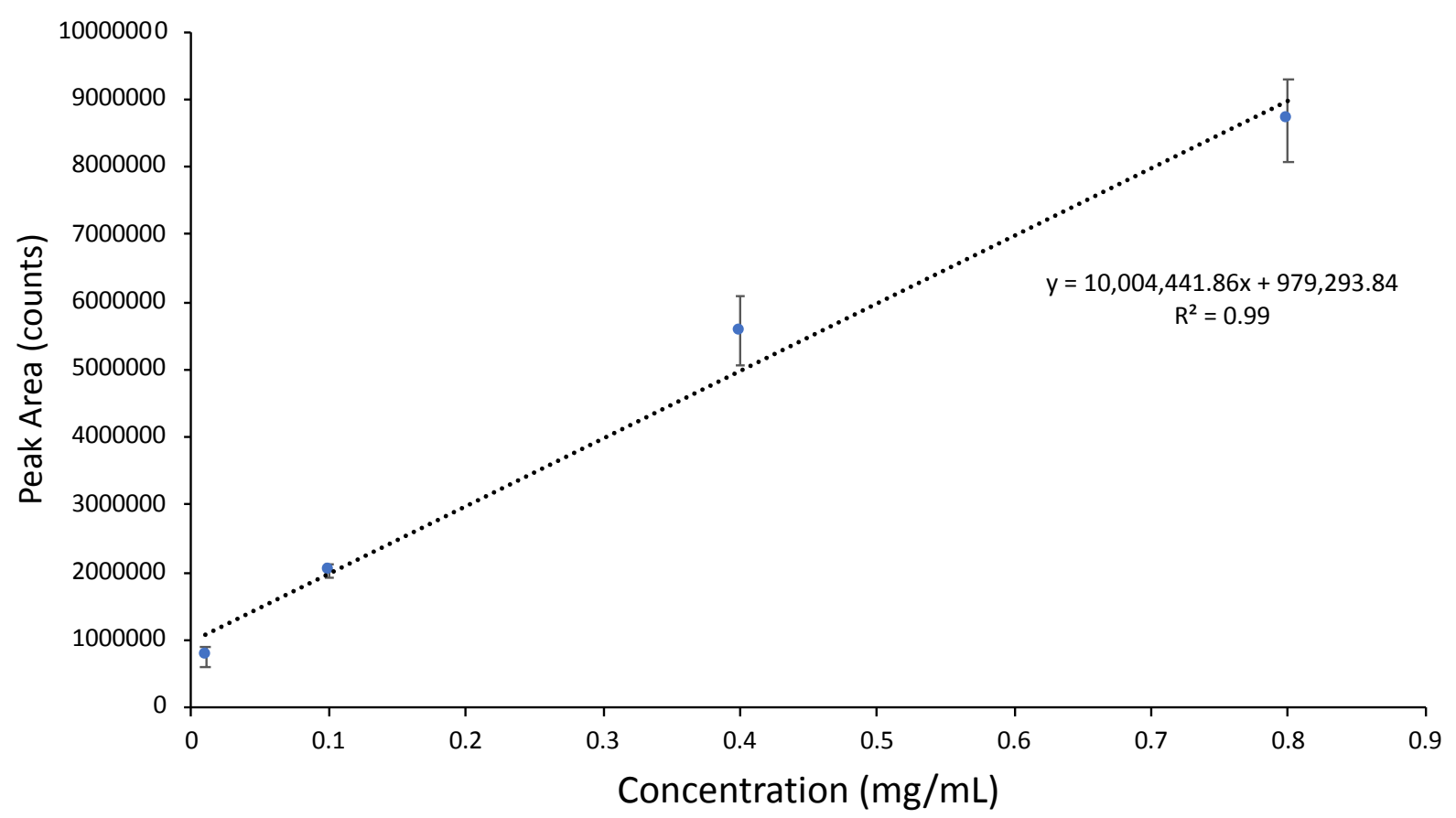

Figure S2. LC-MS calibration curve generated for BSA extraction experiments. The peak area of the +50 charge state $(1329.6 \mathrm{~m} / \mathrm{z})$ of the protein was used. 


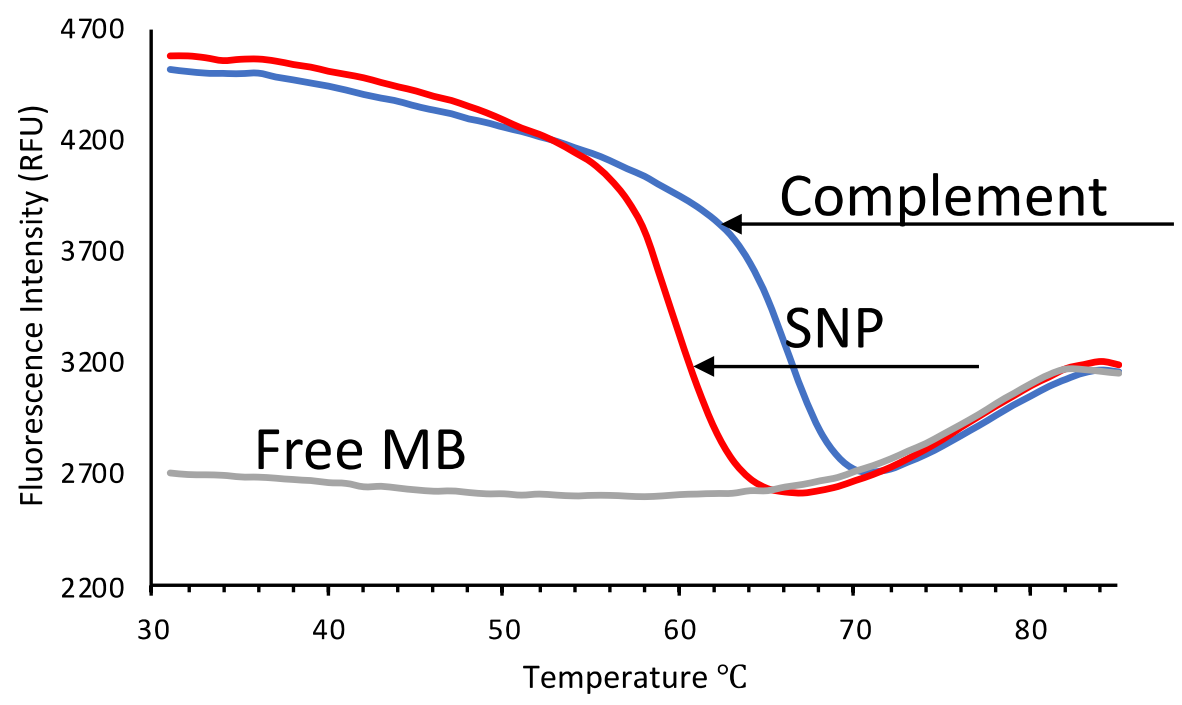

Figure S3. Annealing profiles using MB2 in the presence of the target or a sequence containing a 1 nucleotide mismatch. 

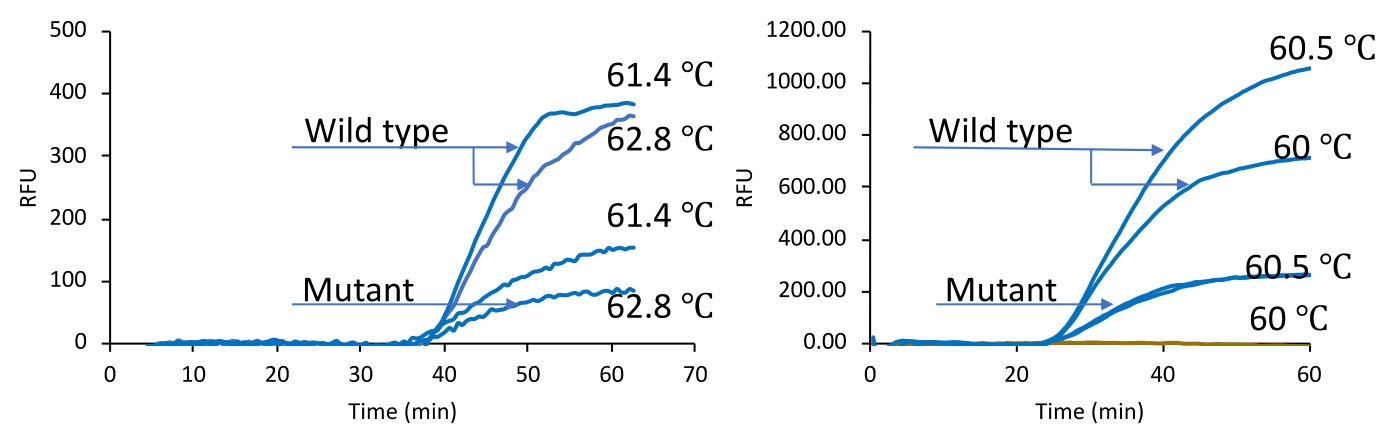

Figure S4. Temperature optimization of the MB-LAMP reaction. Representative real-time amplification plots of the FAM channel when MB-LAMP reactions were performed with 2.34 $\times 10^{5}$ copies of wild type and mutant BRAF V600E. 
A)

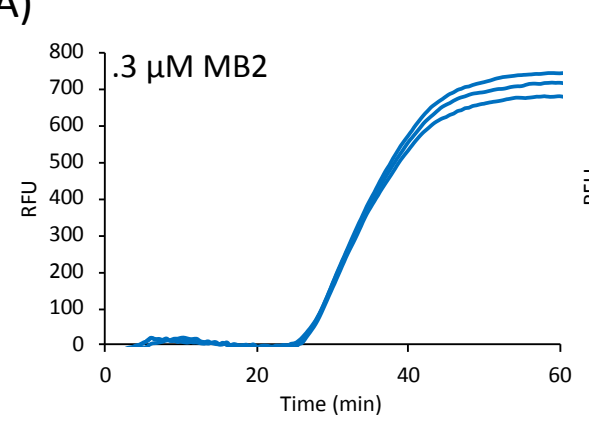

B)

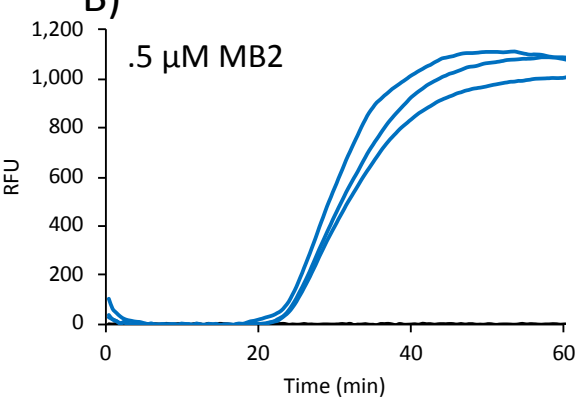

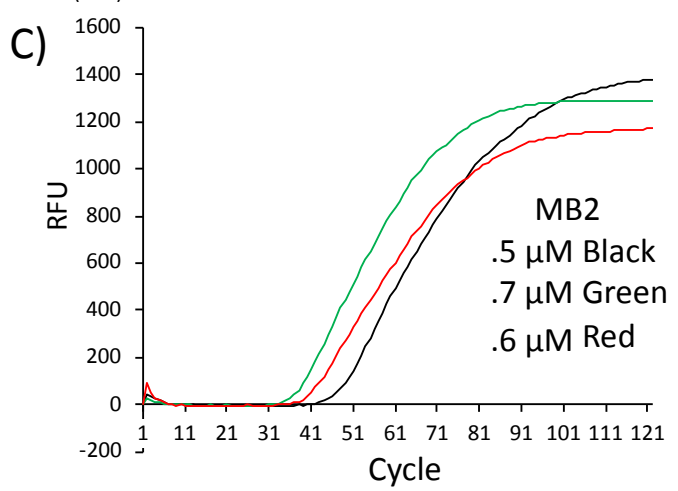

Figure S5. Real-time amplification plots demonstrating the effect of having (A) $0.3 \mu \mathrm{M},(\mathrm{B}, \mathrm{C})$ $0.5 \mu \mathrm{M}$, (C) $0.6 \mu \mathrm{M}$, and (C) $0.7 \mu \mathrm{M}$ of MB2 present in the reaction. 
A)

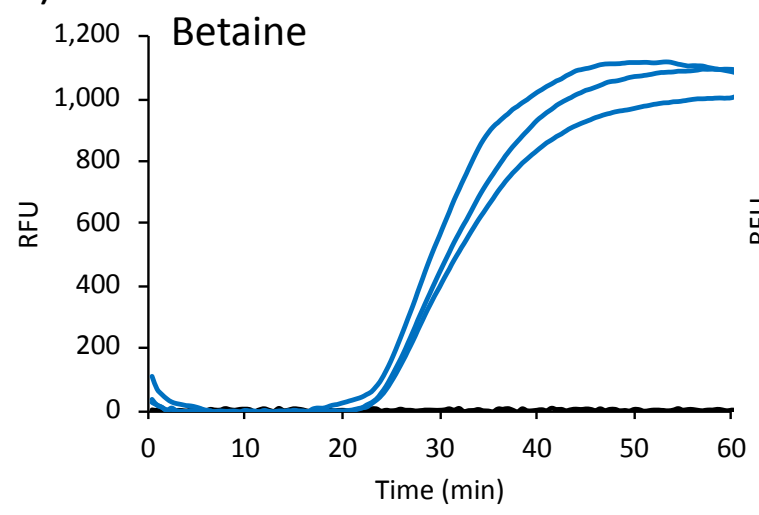

B)

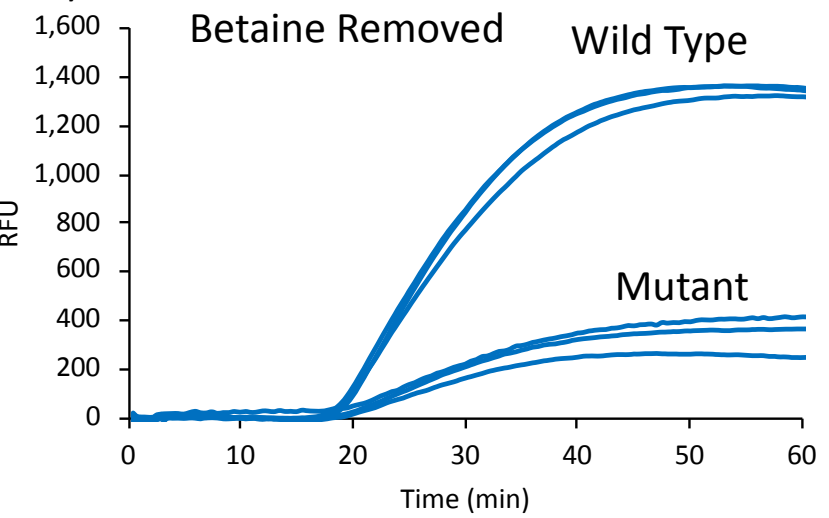

Figure S6. Real-time MB-LAMP amplification using MB2 demonstrating the effect of having betaine present in the MB-LAMP reaction (A) versus removing it from the system (B). 

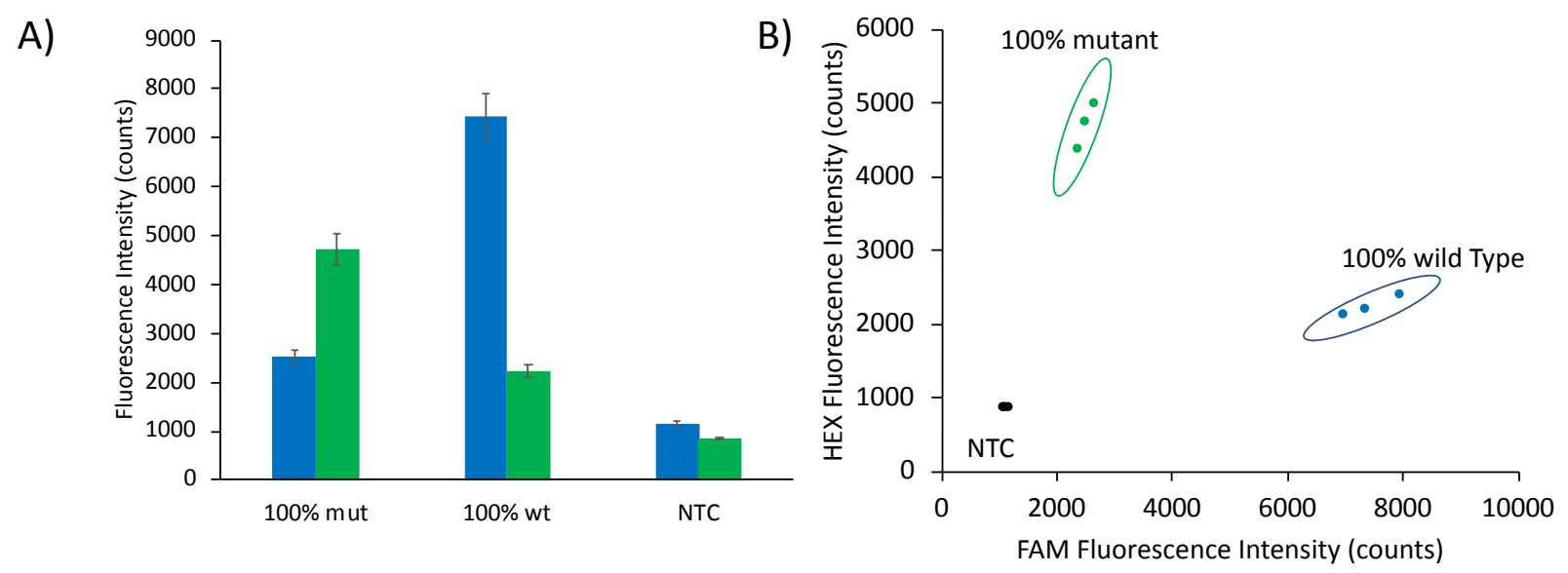

Figure S7. (A) End-point fluorescence measurements of reactions containing 100\% mutant, $100 \%$ wild type, and no template control reactions obtained with a plate reader. (B) Plot of HEX and FAM fluorescence values obtained from each performed reaction. 


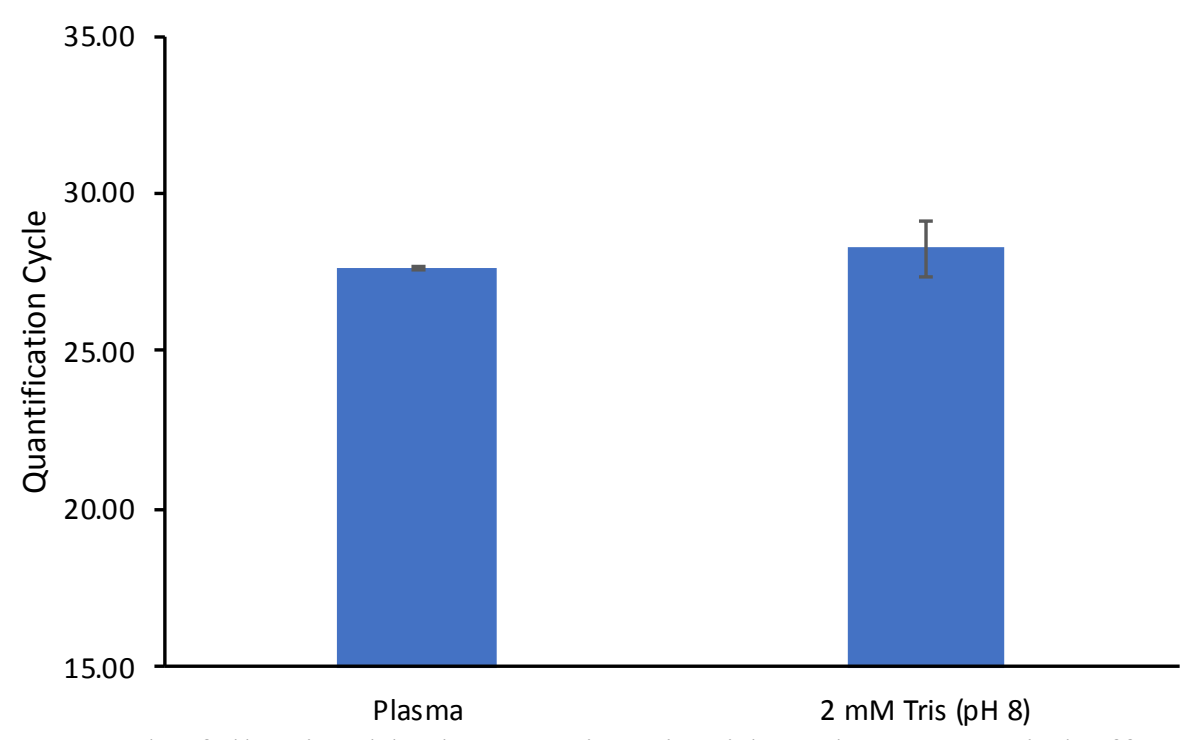

Figure S8. qPCR results following blank extractions in either plasma or Tris buffer. Following the blank extraction and desorption, 10 fg of target DNA was added to the desorption solution. 


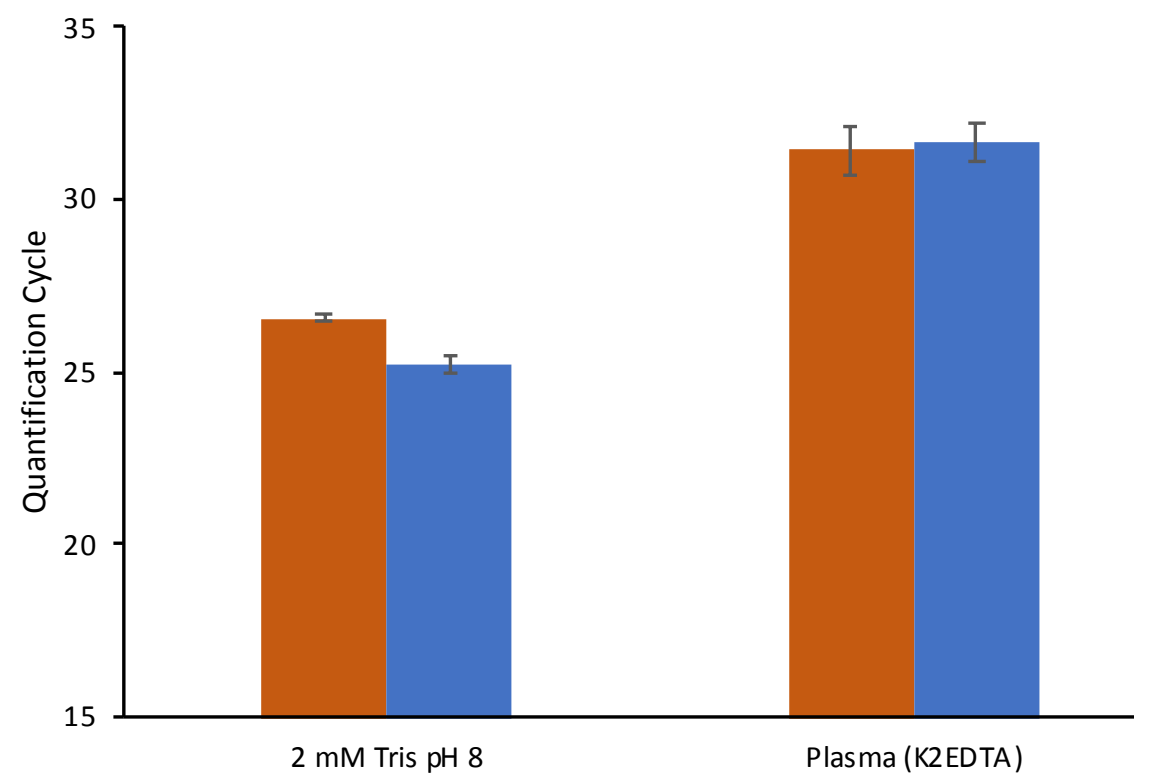

Figure S9. Comparison of the extraction from a $10 \mathrm{pg} \mathrm{mL}^{-1}$ solution of DNA in Tris and plasma using PIL-SPME or silica-coated magnetic beads. The brown columns represent results from the magnetic beads while the blue columns represent the PIL-SPME results. 


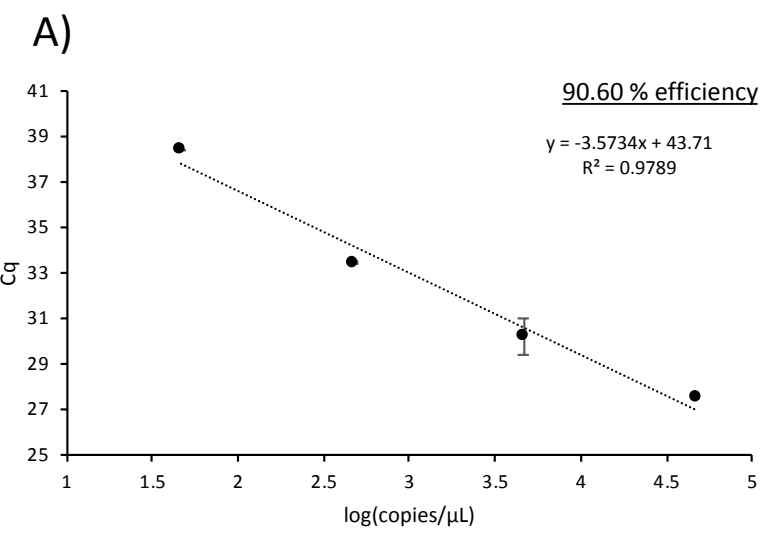

B)

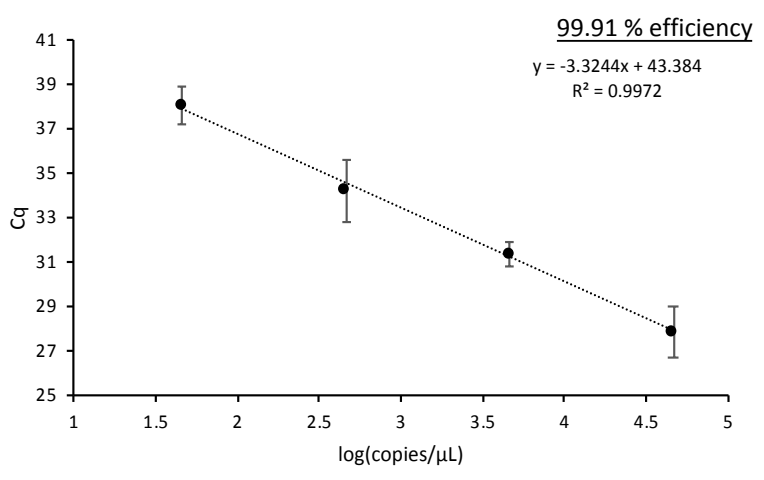

Figure S10. Calibration curves constructed by performing PIL-SPME at 4 different concentration-levels of DNA. Extractions were performed in (A) $40 \mathrm{mg} \mathrm{mL}^{-1}$ and (B) $4 \mathrm{mg} \mathrm{mL}^{-1}$ BSA solutions. Triplicate extractions were performed for each DNA concentration. 